\title{
Channeled spectrum liquid refractometer
}

\author{
A. Gh. Podoleanu, a) S. R. Taplin, D. J. Webb, and D. A. Jackson \\ Applied Optics Group, Physics Laboratory, University of Kent, Canterbury, Kent CT2 7NR, \\ United Kingdom
}

(Received 18 June 1993; accepted for publication 12 July 1993)

\begin{abstract}
We describe an experimental demonstration of a novel technique for liquid refractometry. A channeled spectrum is produced from an optical heam generated by a diode laser operating below threshold by intercepting half of the beam with a liquid cell. The spectrum is analyzed using a grating and a linear CCD array and provides information on the refractive index of the liquid. The experimental results show that accuracies of better than $0.3 \%$ in the index may be obtained with the present method.
\end{abstract}

\section{INTRODUCTION}

The refractive index of liquids is often measured using Abbe or Hilger-Chance refractometers, etc. " There are also methods based on diffraction, ${ }^{2}$ interference, ${ }^{3}$ and the attenuation of light propagating in optical fibers immersed in the liquid. ${ }^{4,5}$ The methods using refractometers are characterized by accuracies of better than $10^{-5}$, but they do not readily lend themselves to automatic real-time monitoring.

Our recent experimental investigation of a channeled spectrum-based displacement sensor ${ }^{6}$ has suggested the possibility of using this technique for the determination or monitoring of refractive index. A channeled spectrum is produced when an interferometer with nonzero path imbalance is illuminated by a broadband (or white light) source. ${ }^{7}$ In the spectral domain, the spacing between the peaks in the spectrum is a function of the path imbalance. In our approach the spectrum is monitored by a linear CCD array after dispersing the light using a diffraction grating.

We have now developed a simple experimental arrangement in which a liquid sample intercepts half of a beam of light from a broadband source; the other half passing through air. After dispersion by a grating the two halves of the beam are recombined on the CCD by a lens.

\section{EXPERIMENTAL ARRANGEMENT}

The experimental configuration is shown in Fig. 1. As a broadband optical source, a multimode laser diode (HL $7611 \mathrm{G}$ ) operating below threshold with a center wavelength of $673 \mathrm{~nm}$ is used. The laser light is collimated and passed through the cell in such a way that half of the beam is intercepted by the liquid while half travels through air. In this way, a simple two-beam interferometric configuration is created. The light is then directed toward a diffraction grating (1200 lines $/ \mathrm{mm}$ ) and the diffaction pattern is examined by a CCD array (Thomson-CSF TH 8711 with TH7931D drive module, 1728 pixels). The temporal and spectral features of the signal are studied using an oscilloscope and a low-frequency spectrum analyzer, respectively. A series of peaks forming the channeled spectrum are ob-

\footnotetext{
a) Permanent address: Physics Department, Technical University "Politechnica," Bucharest, Spl. Independentei 313, 77206, Romania.
}

served within the line shape of the source. The number of peaks and hence the frequency at which these peaks are read out of the CCD is directly proportional to the path imbalance of the two-beam interferometer.

\section{INDEX OF REFRACTION DETERMINATION}

The optical path imbalance in the interferometer, $d$, is given by the relation

$$
d=w\left(n-n_{a}\right),
$$

where $w$ is the width of the cell, $n$ and $n_{a}$ are the indices of refraction of the liquid and air, respectively.

In order to obtain a good signal from the CCD, two conditions must be satisfied: first, the peaks in the spectrum imaged on the CCD must be separated by a distance much larger than the pixel spacing, and second the spectral resolution of the grating must be much better than the free spectral range of the interferometer. Both conditions were satisfied in our experiment.

In this case, it may be shown that the instantaneous frequency of the signal from the CCD, defined as the inverse of the time interval between readout of successive maxima in the channeled spectrum, is approximately given by

$$
\begin{aligned}
f & =\frac{a \cos \theta}{m k F \lambda^{2}} d \\
& =s d \\
& =i\left(n-n_{a}\right),
\end{aligned}
$$

where $a$ is the periodicity of the grating, $\theta$ is the angle between the CCD-grating direction and the normal to the grating, $m$ is the diffaction order, $k$ is the conversion factor of the CCD array and driver (from space to time), $F$ is the focal length of the lens used to display the spectrum over the CCD array, $\lambda$ is the central wavelength of the optical source, and $s$ and $i$ denote the slope of the dependence of frequency on path imbalance and on the index of refraction, respectively.

The cell was filled in turn with several liquids chosen to provide a range of refractive indices and in each case the frequency of the CCD signal was measured using the spectrum analyzer. The results are given in Table I and dis- 


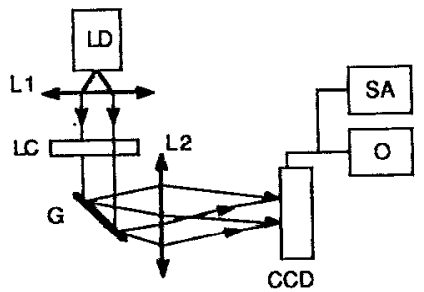

F1G. 1. Experimental arrangement. LD: laser diode; L1, L2: lenses; LC: liquid cell; CCD: CCD array; O: oscilloscope; SA: spectrum analyzer.

played graphically in Fig. 2. The dependence of frequency on index is seen to be linear as expected, and with a slope of

$$
i=\frac{\Delta f}{\Delta n}=19.263 \mathrm{kHz} .
$$

The rms deviation from linearity displayed by these data was $0.3 \%$.

\section{DISCUSSION}

The aim of this article is to report the demonstration of what we believe to be a promising new technique for real time index monitoring. We recognize that many applications will need an accuracy of better than $0.3 \%$ and we have identified a number of sources of error in our simple proof-of-principle experiment which can be eliminated. One error source stems from the orientation of the cell relative to the beam. We needed to take out the cell from the optical bench to clean it before inserting a new liquid. After each change of liquid, on replacing the cell in the beam we tried to find the minimum frequency which corresponds to normal incidence. Nevertheless, the removal of the cell does introduce some error which would be avoided in an on-line system where the liquid would continuously move through the cell.

A second major source of errors is the fact that the values used for the indices of refraction in Table I were quoted for $589.8 \mathrm{~nm}$ (sodium $\mathrm{D}$ line) and not for the center wavelength of our source and were in any case accurate to only 0.001 . Taking into account the dispersion law, the values for $673 \mathrm{~nm}$ should be about $0.2 \%-0.3 \%$ less. ${ }^{7}$ These sources of error coupled with the spectrum

TABLE I. Frequency of the CCD signal for various liquids.

\begin{tabular}{lcc}
\hline \multicolumn{1}{c}{ Liquid } & $n_{D}$ & Frequency $(\mathrm{Hz})$ \\
\hline Methanol & $1.328^{\mathrm{a}}$ & 6334 \\
Water & $1.336^{\mathrm{b}}$ & 6410 \\
Acetone & $1.359^{\mathrm{b}}$ & 6800 \\
Iso-propyl alcohol & $1.375^{\mathrm{c}}$ & 7093 \\
Methyl chloroform & $1.437^{\mathrm{a}}$ & 8398 \\
Benzene & $1.501^{\mathrm{b}}$ & 9625 \\
\hline
\end{tabular}

"Taken from the bottle label.

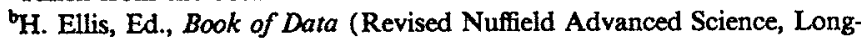
man Group Ltd., 1990), p. 138.

${ }^{\prime} \mathrm{R}$. C. Weast and G. L. Tuve, Eds., Handbook of Chemistry and Physics (Chemical Rubber Co., Cleveland, 1969), p. E-230.

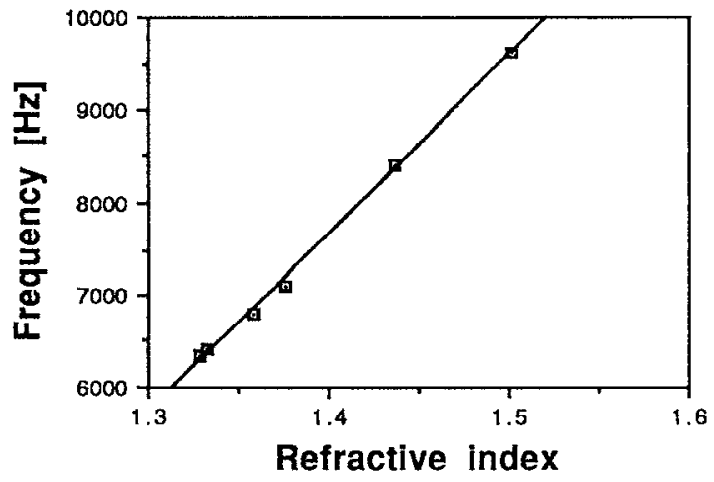

FIG. 2. Frequency of the CCD signal vs index of refraction of the liquid.

analyzer bandwidth of $240 \mathrm{~Hz}$ which determines the accuracy of the frequency measurement may explain a large part of the errors in our simple system.

We believe that by employing a fixed cell and developing dedicated signal processing circuitry the resolution of the system will be better than $0.1 \%$.

\section{CONCLUSIONS}

A technique for the determination of the index of refraction of liquids is described. The arrangement uses a CCD, a grating, and a multimode laser operating below threshold. The scheme has the advantage that the refractive index of the liquid may be monitored in real time, at a rate determined by the CCD readout time, which was $7 \mathrm{~ms}$ in our case but could be faster. This makes the technique suitable for dynamic process control. In addition, the technique may be very easily put into practice and the alignment is not critical. The system has no moving part and is unaffected by variations in the liquid absorption. The method is also suitable for the determination of the index of refraction of plane parallel sheets of transparent materials or alternatively for the determination of the width of these materials if their index is known. Further studies are needed to determine the ultimate attainable performance of the technique.

\section{ACKNOWLEDGMENTS}

Adrian Podoleanu acknowledges the support of the Commission of the European Communitics within the framework of the TEMPUS scheme. Steve Taplin acknowledges the support of Shell U.K. The authors appreciate the help of Dr. Bernard Hurle in the construction of the liquid cell.

${ }^{1}$ S. Longhurst, Geometrical and Physical Optics, 3rd ed. (Longmans, London, 1962), p. 132.

${ }^{2}$ A. A. Zaidi, Rev. Sci. Instrum. 59, 1153 (1988).

${ }^{3}$ D. J. Webb, R. P. Tatam, and D. A. Jackson, Rev. Sci. Instrum. 60, 3347 (1989).

${ }^{4}$ M. S. Meyer and G. L. Eesley, Rev. Sci. Instrum. 58, 2047 (1987).

${ }^{5}$ A. L. Harmer, in Proceedings of the First International Conference on Optical Fibre Sensors, IEEE Conf. Publ. (London) 221, 104 (1983).

${ }^{6}$ S. Taplin, A. Gh. Podoleanu, D. J. Webb, and D. A. Jackson, Electron. Lett. 29, 896 (1993).

${ }^{7}$ F. A. Jenkins and H. E. White, Fundamentals of Optics (McGrawHill, New York, 1983), p. 311. 